\title{
Automatic Tissue Segmentation with Deep Learning in Patients with Congenital or Acquired Distortion of Brain Anatomy
}

\author{
Gabriele Amorosino ${ }^{1,2}$, Denis Peruzzo ${ }^{3}$, Pietro Astolfi ${ }^{1,4}$, Daniela Redaelli ${ }^{3}$, \\ Paolo Avesani ${ }^{1,2}$, Filippo Arrigoni ${ }^{3}$ and Emanuele Olivetti ${ }^{1,2}$ \\ 1 NeuroInformatics Laboratory (NILab), Bruno Kessler Foundation, Trento, Italy \\ 2 Center for Mind and Brain Sciences (CIMeC), University of Trento, Italy \\ ${ }^{3}$ Neuroimaging Lab, Scientific Institute IRCCS Eugenio Medea, \\ Bosisio Parini (Lecco), Italy \\ 4 PAVIS, Italian Institute of Technology (IIT), Genova, Italy \\ olivetti@fbk.eu gamorosino@fbk.eu
}

\begin{abstract}
Brains with complex distortion of cerebral anatomy present several challenges to automatic tissue segmentation methods of T1-weighted MR images. First, the very high variability in the morphology of the tissues can be incompatible with the prior knowledge embedded within the algorithms. Second, the availability of MR images of distorted brains is very scarce, so the methods in the literature have not addressed such cases so far. In this work, we present the first evaluation of state-of-theart automatic tissue segmentation pipelines on T1-weighted images of brains with different severity of congenital or acquired brain distortion. We compare traditional pipelines and a deep learning model, i.e. a 3D U-Net trained on normal-appearing brains. Unsurprisingly, traditional pipelines completely fail to segment the tissues with strong anatomical distortion. Surprisingly, the 3D U-Net provides useful segmentations that can be a valuable starting point for manual refinement by experts/neuroradiologists.
\end{abstract}

\section{Introduction}

Accurate segmentation of brain structural MR images into different tissues, like white matter (WM), gray matter (GM) and cerebrospinal fluid (CSF), is of primary interest for clinical and neuroscientific applications, such as volume quantification, cortical thickness analysis and bundle analysis.

Since manual segmentation of the brain tissues is extremely time consuming it is usually performed by means of well-established automated tools, such as FSL [7, SPM [1, FreeSurfer [5] and ANTs [12. Typically, these tools obtain excellent quality of segmentation in normal-appearing brains.

More recently, brain tissue segmentation has been addressed by deep learning algorithms like convolutional neural networks (CNNs) 9]10]4[13], applied directly to $\mathrm{T} 1$ or $\mathrm{T} 2$ weighted MR images. The quality of segmentation obtained 
by such methods is again excellent and the computational cost, once trained, is usually greatly reduced with respect to traditional pipelines.

Especially in children, many congenital (e.g. malformations, huge arachnoid cysts) or acquired (e.g. severe hydrocephalus, encephalomalacia due to perinatal injuries) conditions can cause complex modifications of cerebral anatomy that alter the structural and spatial relationship among different brain structures. Automatically segmenting such brains presents multiple challenges mainly due to the high variability of the morphology together with the scarce availability of data. Moreover, the prior knowledge encoded in automated pipelines, or the set of images used to train segmentation algorithms, do not cover such cases.

In this work, for the first time, we present results of different well-established brain tissue segmentation pipelines on T1 images of malformed and highly distorted brains in the pediatric age. Unsurprisingly, we observe that the quality of segmentation is highly variable with traditional pipelines, and it fails when the complexity of the brain distortion and the severity of the malformation are high.

Moreover, as a major contribution, we show the results of a CNN for segmentation of medical images, namely the 3D U-Net 3[11, trained on over 800 pediatric subjects with normal brain. Surprisingly, the 3D U-Net segments brains with moderate and severe distortion of brain anatomy either accurately or at least to a sufficient level to consider manual refinement by expert radiologists.

The evaluation study of the U-Net presented here is first conducted on a large sample of normal brain images, as a sanity check, to quantitatively assess that the specific implementation and training procedure reaches state-of-the-art quality of segmentation. The evaluation on distorted brains is instead qualitative, because of the small sample available due to the rarity of the condition, as well as the current lack of gold standard segmentation.

If confirmed by future and more extensive studies, this result opens the way to CNNs-based tissue segmentation methods in applications, even in the case of malformed and highly distorted brains. From the methodological point of view, CNNs show a much higher degree of flexibility in tissue segmentation than well-established pipelines, despite being trained on segmentations obtained from those same pipelines.

\section{Materials}

We assembled a dataset of over $900 \mathrm{MR}$ images from subjects and patients in the pediatric age, divided into two parts: normal brains and distorted brains. The first part consists of $570 \mathrm{~T} 1$-w images from public databases $\left(\mathrm{C}-\mathrm{MIND}^{5}\right.$. NIMH $\left.{ }^{6}\right)$ and 334 T1-w images acquired in-house by the authors during clinical activity at IRCCS Eugenio Medea (Italy). The second part comprises 21 patients, again acquired at IRCCS E.Medea.

5 https://research.cchmc.org/c-mind NIH contract \#s HHSN275200900018C.

6 http://pediatricmri.nih.gov 


\section{$2.1 \quad$ Normal Brains}

- Public Databases.

- T1-w images from 207 healthy subjects of the C-MIND database (165 from CCHMC site, with average age $8.9(\mathrm{SD}=5.0)$ and 42 from UCLA site, with average age $7.6(\mathrm{SD}=3.8)$ ) both with 3D MPRAGE MRI 3T scan sequence $\left(\mathrm{TR}=8000 \mathrm{~ms}, \mathrm{TE}=3.7 \mathrm{~ms}\right.$, Flip angle $=8^{\circ}, \mathrm{FOV}=256 \times 224 \times 160$, voxel spacing $=1 \times 1 \times 1 \mathrm{~mm}^{3}$ ).

- T1-w images from 363 healthy subjects (average age $10.7(\mathrm{SD}=6.0)$ ) from the Pediatric MRI database of NIMH Data Archive [6], MRI 1.5T scanner with two different sequences:

* 284 subjects acquired with a 3D T1-w Sequence 3D RF-spoiled gradient echo sequence (TR $22-25 \mathrm{~ms}$, TE $=10-11 \mathrm{~ms}$, Excitation pulse $=30^{\circ}$, Refocusing pulse $180^{\circ}, \mathrm{FOV}=160-180 \times 256 \times 256$, voxel spacing $=1 \times 1 \times 1 \mathrm{~mm}^{3}$ )

* 79 subjects acquired with a T1-w Sequence Spin echo $(\mathrm{TR}=500$ $\mathrm{ms}, \mathrm{TE}=12 \mathrm{~ms}$, Flip angle $=90^{\circ}$, Refocusing pulse $=180^{\circ}$ Field of view $=192 \times 256 \times 66$, voxel spacing $=1 \times 1 \times 3 \mathrm{~mm}^{3}$ )

- In-House Database: IRCCS E.MEDEA. T1-w images from 334 subjects with normal brain, acquired in-house with average age $10.6(\mathrm{SD}=5.2)$ and 3D T1-w MPRAGE 3T MRI scan sequence $(\mathrm{TR}=8000 \mathrm{~ms}$, TE $=4 \mathrm{~ms}$, Flip angle $=8^{\circ}, \mathrm{FOV}=256 \times 256 \times 170$, voxel spacing $\left.=1 \times 1 \times 1 \mathrm{~mm}^{3}\right)$.

\subsection{Distorted Brains}

Images from two groups of patients acquired in-house at IRCSS E.Medea with the same MR and scan sequence described above. In detail:

- Agenesis of Corpus Callosum. 12 patients with agenesis of corpus callosum $(\mathrm{ACC})$ (average age $5.8(\mathrm{SD}=5.2))$. Callosal agenesis is characterized by colpocephaly, parallel ventricles, presence of Probst bundles and upward extension of third ventricle. See some cases in Figure 1.

- Complex distortions. 9 patients (average age $7.9(\mathrm{SD}=3.2)$ ) with severe parenchymal distortion related to complex malformations (4 cases), parenchymal poroencephalic lesions and severe hydrocephalus (4 cases) and massive arachnoid cyst (1 case). See some cases in Figure 2 .

\subsection{Pre-processing and Reference Tissue Segmentation}

All T1-w images received bias field correction (N4BiasFieldCorrection) and AC-PC alignment. The reference segmentation of normal brains was performed with the AntsCorticalThickness.sh script of ANTs [12] with the PTBP (pediatric) prior [2, resulting in a 3D mask with 7 labels (6 tissues): background, Cerebrospinal fluid (CSF), Gray matter (GM), White matter (WM), Deep gray matter (DGM), Trunk and Cerebellum. The results of the segmentations were visually assessed by two experts in pediatric neuroimaging. 


\section{Methods}

A standard whole-brain 3D U-Net model 311 was implemented to predict the brain masks of 6 tissues plus background from T1-w images. The architecture of the network consists of 3 main parts: the contraction path, the bottleneck and the expansion path. The input and the output layer are for $256 \times 256 \times 256$ isotropic 3D images, resolution to which all input images are initially resampled. The adopted architecture, described below, has minor changes with respect to the literature to reduce the memory footprint and to fit the GPU used during the experiments reported in Section 4.

The contraction path consists of 4 blocks, each with two convolutional layers (3x3x3 kernel followed by ReLU) followed by MaxPooling (2x2x2 filter, stride $2 \times 2 \times 2)$ for downsampling. The size of the downsampling over the blocks is $256 \rightarrow 128 \rightarrow 64 \rightarrow 32 \rightarrow 16$, while the application of convolutional layers produces an increasing number of feature maps over the blocks, $12 \rightarrow 24 \rightarrow 48 \rightarrow 96$. The bottleneck consists of 2 convolutional layers both of 192 filters $(3 \times 3 \times 3$ kernel then ReLU), followed by a dropout layer (dropout rate: 0.15 ) used to prevent overfitting. Similarly to the contraction path, the expansion path consists of 4 blocks, each with transposed convolution for upsampling, followed by concatenation with contraction features (skip connected) and two convolutions. The upsampling size over the blocks is the opposite of the downsampling, i.e. from 16 to 256 . All convolutional layers have $3 \times 3 \times 3$ kernel then ReLU, that produce a decreasing number of feature maps from 192 to 12 .

Multiclass classification (6 tissues and background), is obtained with a last layer of convolution with 7 1x1x1 filters, followed by a softmax activation.

\section{Experiments}

We qualitatively compared the segmentation pipelines of FSL, FreeSurfer, SPM, ANTs and the 3D U-Net on T1-weighted images from patients with agenesis of corpus callosum (ACC) and with complex distortions of brain anatomy, as described in Section 2.2 Different pipelines produced different sets of segmented tissues. To harmonize the results, we considered only 3 main tissues: gray matter (GM), white matter (WM) and the cerebrospinal fluid (CSF). These tissues are the ones of greater interest in most of the applications of brain tissue segmentation. Moreover, for the pipelines segmenting the deep gray matter (DGM), we labeled DGM as GM. The technical details of each pipeline are the following:

- FSL v6 [7]: we used fsl_anat $7^{7}$ with default parameter values.

- FreeSurfer v6 [5]: we used recon-ald 8 with default parameter values.

- SPM12 11: we used default parameter values and default prior.

- ANTs v2.2.0 [12]: we used AntsCorticalThickness.sh with default parameter vales and the PTBP (pediatric) prior 2. We considered only the steps till tissue segmentation.

7 https://fsl.fmrib.ox.ac.uk/fsl/fslwiki/fsl_anat

8 https://surfer.nmr.mgh.harvard.edu/fswiki/recon-all 
- 3D U-Net. The model was implemented with TensorFlow 1.8.0 [8] trained on T1-weighted images from the Normal Brains Datasets of $\approx 900$ images described in Section 2. We kept apart 90 randomly selected images for a sanity check, i.e., to assess that the trained model could reach state-of-theart quality of segmentation on healthy subjects. The training (loss: crossentropy, Adam optimization, learning rate: $10^{-4}$ ) was performed iteratively, one image at a time taken at random from the training set, looping over the whole set for 60 epochs.

All computations were performed on a dedicated workstation: 6 cores processor Intel(R) Xeon(R) CPU E5-1650 v4 3.60GHz, 128Gb RAM, GPU: NVIDIA GeForce GTX 1080TI 11Gb RAM.

\subsection{Results}

Except for FreeSurfer (recon-all), all pipelines carried out all the required segmentations. FreeSurfer failed in all cases of severe anatomical distortion. Specifically, recon-all did not converge during either Talairach registration or skull stripping. SPM completed the segmentation task in all subjects. However, its confidence in the prior for the segmentation initialization and optimization leads to many major macroscopic errors. Given the strong limits on the length of this article, we do not illustrate and discuss the uninteresting results of FreeSurfer and SPM.

In Figure 1, we show a paradigmatic set of axial slices segmented by FSL, ANTs and the $3 \mathrm{D}$ U-Net, from patients with ACC, i.e. from 4 of the 12 subjects described in Section 2.2. Similarly, in Figure 2, we report paradigmatic axial slices segmented by those methods, from 4 of the subjects with complex cerebral distortions. For the segmentations of all methods, we re-labeled as CSF all the voxels inside the brain mask of each patient that were incorrectly segmented as background. In both figures in the last row, for each subject, we highlight a detail of the slice for one of the segmentation methods (indicated with a dashed square, above in the same column). Such details are discussed in Section 5

Finally, in Table 1, we report the results of the sanity check, i.e. that the training process of the $3 \mathrm{D}$ U-Net on normal brains was successful. The numbers represent the average quality of segmentation (DSC, Dice similarity coefficient) obtained by the 3D U-Net for the reference segmentation of the 6 tissues of normal brains described in Section 2.3. These results are comparable to those in the state-of-the-art 4113.

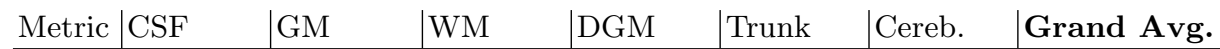
\begin{tabular}{l|l|l|l|l|l|l|l}
\hline DSC & $0.87 \pm 0.06$ & $0.95 \pm 0.04$ & $0.95 \pm 0.03$ & $0.93 \pm 0.02$ & $0.93 \pm 0.03$ & $0.96 \pm 0.02$ & $0.93 \pm 0.03$ \\
\hline
\end{tabular}

Table 1. 3D U-Net: average Dice similarity coefficient (DSC) for segmentations of 6 tissues, plus grand average, on 90 normal brains, after 60 epochs of training. 


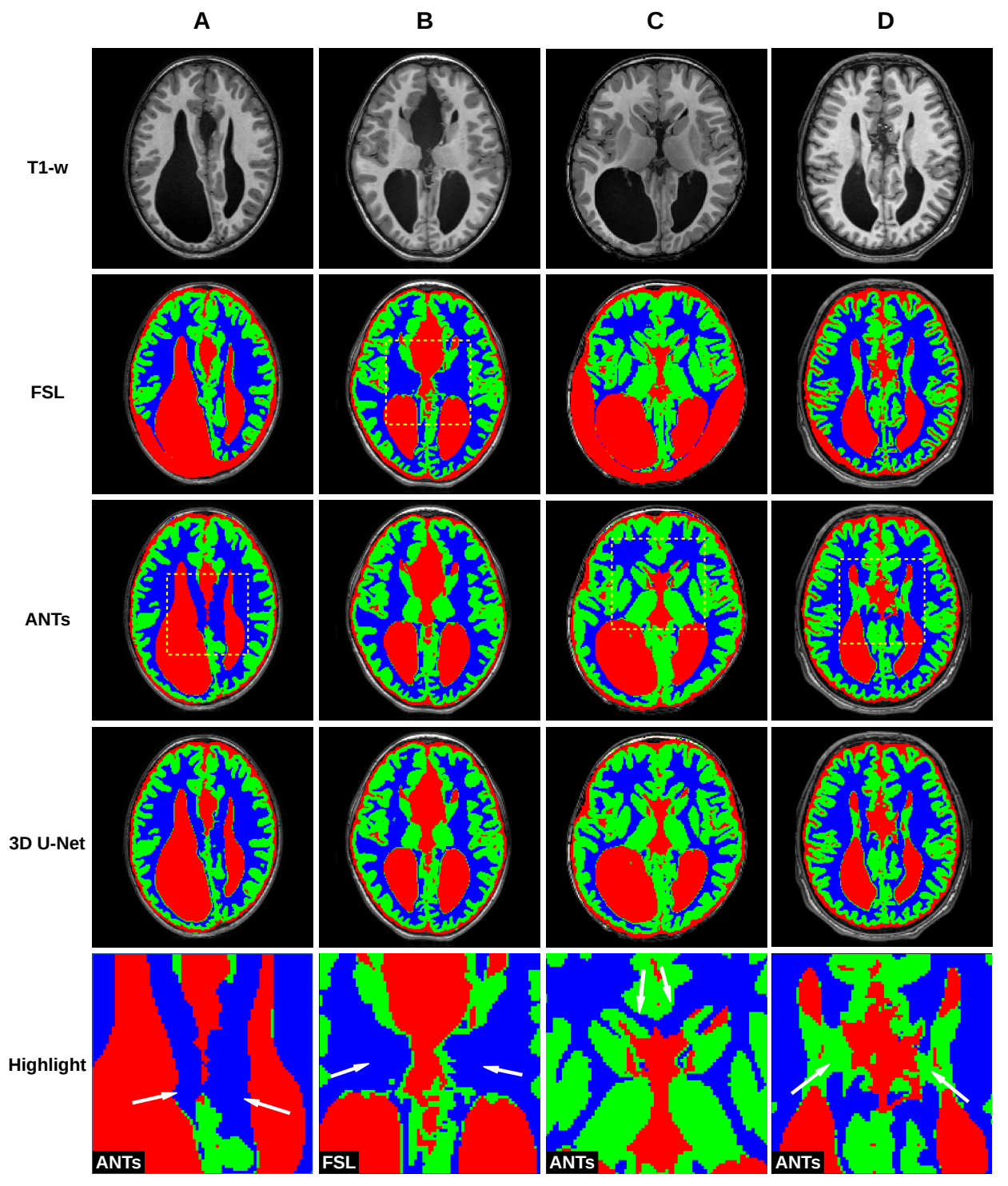

Fig. 1. First row: T1-weighted MR images of 4 subjects (A, B, C and D) with agenesis of corpus callosum. Below, the related tissue segmentations (GM in green, WM in blue and CSF in red) of the following pipelines: FSL (2nd row), ANTs (3rd row) and 3D U-Net (4th row). In the 5th row, for each subject, we show the enlarged view of one of the segmentations, indicated above with a dashed yellow square. White arrows point to the highlights discussed in Section 5 


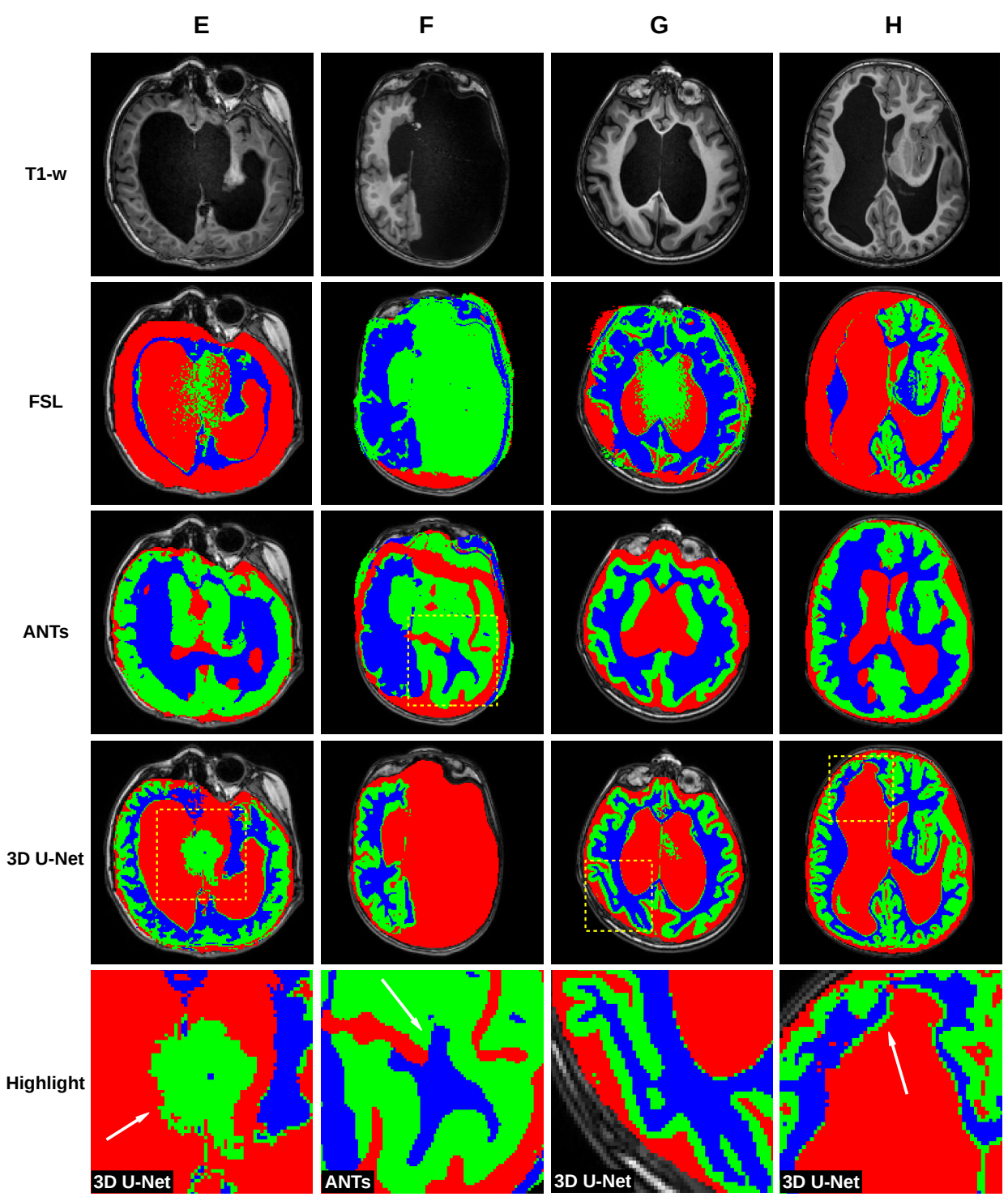

Fig. 2. First row: T1-weighted MR images of 4 subjects (E, F, G and H) with complex cerebral distortions. Below, the related tissue segmentations (GM in green, WM in blue and CSF in red) of the following pipelines: FSL (2nd row), ANTs (3rd row) and 3D U-Net (4th row). In the 5th row, for each subject, we show the enlarged view of one of the segmentations, indicated above with a dashed yellow square. White arrows point to the highlights discussed in Section 5 


\section{Discussion}

Figure 1 shows that FSL fails to segment GM and WM in cases of moderate and severe ventricular dilatation with thinning of the WM (case A, C and less evident in D). In one case (B) FSL also misses identifying the thalami as (deep) GM. ANTs performs better in identifying (deep) GM and the cortex at the convexity. However, case $\mathrm{A}$ and $\mathrm{C}$ show that it may fail in differentiate between GM and subcortical WM on the mesial surface of the hemispheres. This may be related to the prior used by that pipeline, which is based on the anatomy of normal subjects and not designed to recognize spatial reorganization of the cortex, especially in the midline, like in ACC cases. A similar error is in case $\mathrm{C}$ where a cortical component close to the head of the caudate is misclassified as WM. Finally in D, Probst bundles, which are abnormal WM tracts running parallel to the medial ventricular wall, are labelled as GM. In contrast, the 3D U-Net performs well in segmenting ACC. The most relevant error in these cases is at the interface between ventricles and WM: the 3D U-Net wrongly identifies a very thin layer of GM along the inner ventricular surface. This is probably related to partial volume effects.

Figure 2 shows that, in case of complex malformations and severe parenchymal distortion, FSL and ANTs are unreliable and incur in major macroscopic errors, as opposed to 3D U-Net which performs vastly better. In cases of severe ventricular dilatation and distortion $(\mathrm{E}$ and $\mathrm{H})$, FSL fails to segment the cortex, which is wrongly labelled as CSF. In case F, the CSF collection that replaces the left hemisphere is misclassified as the cortex. In G, FSL fails to properly segment the pachygyric (i.e. with a reduced number of gyri) cortex. Finally, some intensity inhomogeneities in the deep ventricular CSF are misclassified as GM (E and G). With ANTs, which is based on priors, the pipeline is forced to segment WM, GM and CSF in the missing hemisphere (F) or when the anatomy is highly irregular. In all of these cases, the pipeline misplaces structures (E, G, H) or segment structures that are actually missing (F). The 3D U-Net outperforms FSL and ANTs in all cases (e.g. G), with few mistakes. The main issues are: i) the mislabeling of signal inhomogeneities in the deep CSF (E, same as FSL), and the segmentation of a subtle layer of GM at the border between lateral ventricles and WM (H, as done in ACC cases). Care must be used in evaluating CSF/WM interface in cases of brain malformations because, at this level, heterotopic GM nodule may occur.

\subsection{Conclusions}

In this work, we observe a much higher accuracy of the 3D U-Net when segmenting brains with different degrees of anatomical distortion, compared to wellestablished pipelines. This is surprising given that such cases were not used in the training phase. At the same time, the 3D U-Net can reproduce the high quality of segmentation of ANTs on normal brains. Clearly, the results on distorted brains are not perfect but still a valuable starting point for manual refinement 
by experts/neuroradiologists. In future work we plan to manually segment T1$\mathrm{w}$ images of the distorted brains, to create a gold standard and to be able to quantify the quality of segmentation of the 3D U-Net and to use some of them during the training process of the network.

\section{Acknowledgment}

Data used in the preparation of this article were obtained from the C-MIND Data Repository created by the C-MIND study of Normal Brain Development. This is a multisite, longitudinal study of typically developing children from ages newborn through young adulthood conducted by Cincinnati Children's Hospital Medical Center and UCLA and supported by the National Institute of Child Health and Human Development (Contract \#s HHSN275200900018C). A listing of the participating sites and a complete listing of the study investigators can be found at https://research.cchmc.org/c-mind. This manuscript reflects the views of the authors and may not reflect the opinions or views of the NIH. 


\section{References}

1. Ashburner, J., Friston, K.J.: Voxel-based morphometry-the methods. NeuroImage 11(6 Pt 1), 805-821 (Jun 2000), http://dx.doi.org/10.1006/nimg.2000.0582

2. Avants, B., ntustison, Wang, D.J.: The Pediatric Template of Brain Perfusion (PTBP) (Feb 2015), https://figshare.com/articles/The_Pediatric_ Template_of_Brain_Perfusion_PTBP_/923555

3. Çiçek, Ö., Abdulkadir, A., Lienkamp, S.S., Brox, T., Ronneberger, O.: 3D U-Net: Learning Dense Volumetric Segmentation from Sparse Annotation. In: Ourselin, S., Joskowicz, L., Sabuncu, M.R., Unal, G., Wells, W. (eds.) Medical Image Computing and Computer-Assisted Intervention - MICCAI 2016, vol. 9901, pp. 424-432. Springer International Publishing, Cham (2016), http://link.springer . com/10. 1007/978-3-319-46723-8_49

4. Cullen, N.C., Avants, B.B.: Convolutional Neural Networks for Rapid and Simultaneous Brain Extraction and Tissue Segmentation. In: Spalletta, G., Piras, F., Gili, T. (eds.) Brain Morphometry, vol. 136, pp. 13-34. Springer New York, New York, NY (2018), http://link.springer.com/10.1007/978-1-4939-7647-8_2

5. Dale, A.M., Fischl, B., Sereno, M.I.: Cortical surface-based analysis. I. Segmentation and surface reconstruction. NeuroImage 9(2), 179-194 (Feb 1999)

6. Evans, A.C.: The NIH MRI study of normal brain development. NeuroImage 30(1), 184-202 (Mar 2006), https://linkinghub.elsevier.com/retrieve/pii/ S105381190500710X

7. Jenkinson, M., Beckmann, C.F., Behrens, T.E.J., Woolrich, M.W., Smith, S.M.: FSL. NeuroImage 62(2), 782-790 (2012), http://www.sciencedirect.com/ science/article/pii/S1053811911010603

8. Martín Abadi, Ashish Agarwal, Paul Barham, Eugene Brevdo, Zhifeng Chen, Craig Citro, Greg S. Corrado, Andy Davis, Jeffrey Dean, Matthieu Devin, Sanjay Ghemawat, Ian Goodfellow, Andrew Harp, Geoffrey Irving, Michael Isard, Jia, Y., Rafal Jozefowicz, Lukasz Kaiser, Manjunath Kudlur, Josh Levenberg, Dandelion Mané, Rajat Monga, Sherry Moore, Derek Murray, Chris Olah, Mike Schuster, Jonathon Shlens, Benoit Steiner, Ilya Sutskever, Kunal Talwar, Paul Tucker, Vincent Vanhoucke, Vijay Vasudevan, Fernanda Viégas, Oriol Vinyals, Pete Warden, Martin Wattenberg, Martin Wicke, Yuan Yu, Xiaoqiang Zheng: TensorFlow: Large-Scale Machine Learning on Heterogeneous Systems (2015), https://www.tensorflow.org/

9. Moeskops, P., Viergever, M.A., Mendrik, A.M., de Vries, L.S., Benders, M.J.N.L., Išgum, I.: Automatic Segmentation of MR Brain Images With a Convolutional Neural Network. IEEE Transactions on Medical Imaging 35(5), 1252-1261 (May 2016)

10. Rajchl, M., Pawlowski, N., Rueckert, D., Matthews, P.M., Glocker, B.: NeuroNet: Fast and Robust Reproduction of Multiple Brain Image Segmentation Pipelines (Apr 2018), https://openreview.net/forum?id=Hks1TRisM

11. Ronneberger, O., Fischer, P., Brox, T.: U-Net: Convolutional Networks for Biomedical Image Segmentation. In: Navab, N., Hornegger, J., Wells, W.M., Frangi, A.F. (eds.) Medical Image Computing and Computer-Assisted Intervention - MICCAI 2015. pp. 234-241. Lecture Notes in Computer Science, Springer International Publishing (2015)

12. Tustison, N.J., Cook, P.A., Klein, A., Song, G., Das, S.R., Duda, J.T., Kandel, B.M., van Strien, N., Stone, J.R., Gee, J.C., Avants, B.B.: Large-scale evaluation of ANTs and FreeSurfer cortical thickness measurements. NeuroImage 99, 166-179 (Oct 2014) 
13. Yogananda, C.G.B., Wagner, B.C., Murugesan, G.K., Madhuranthakam, A., Maldjian, J.A.: A Deep Learning Pipeline for Automatic Skull Stripping and Brain Segmentation. In: 2019 IEEE 16th International Symposium on Biomedical Imaging (ISBI 2019). pp. 727-731 (Apr 2019), iSSN: 1945-7928

\section{Supplementary Materials}

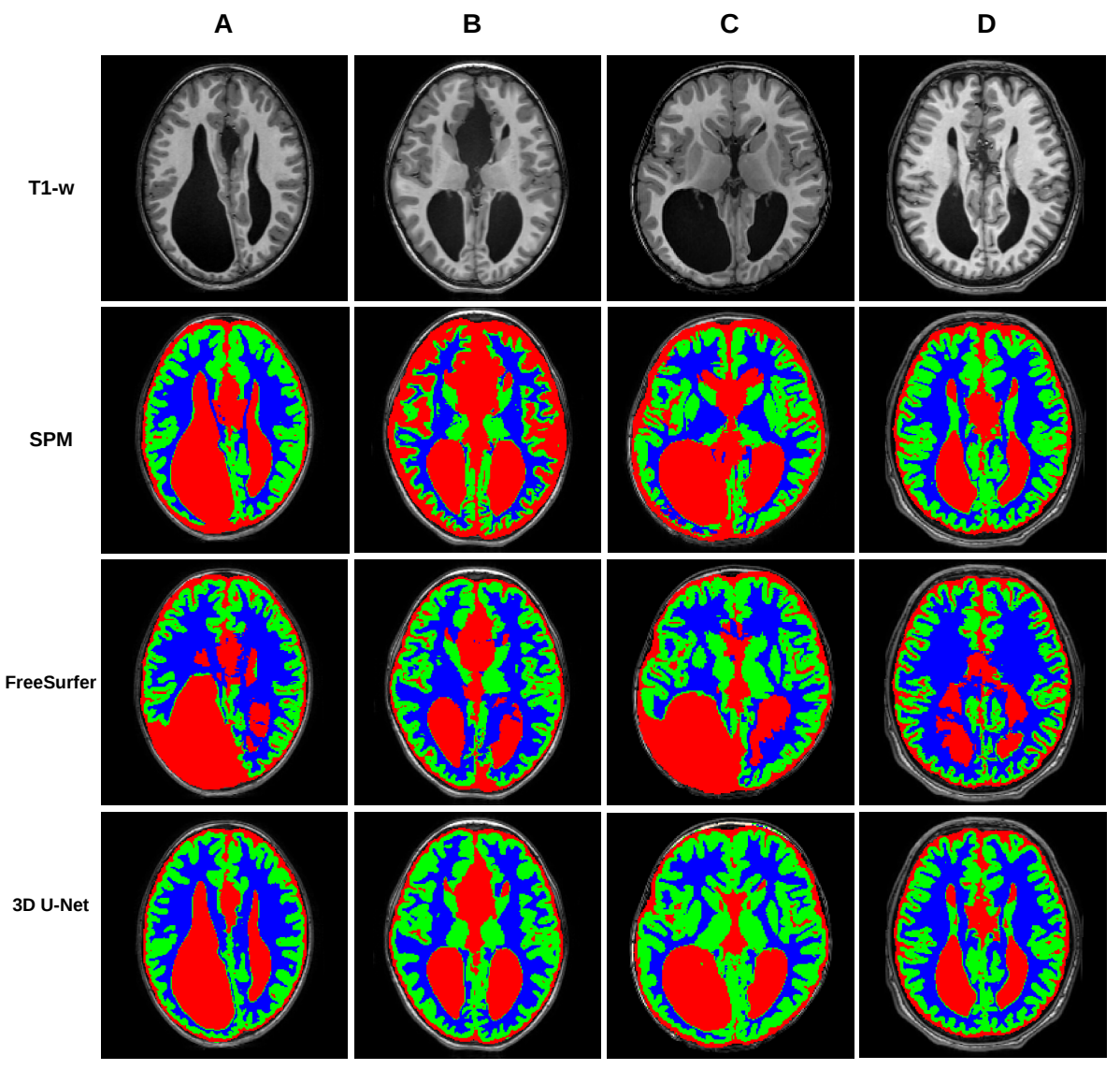

This is an extension of Figure 1, i.e. paradigmatic segmentations of patients with Agenesis of Corpus Callosum, where we show SPM and FreeSurfer that we excluded in the manuscript, together with the 3D U-Net for comparison. The segmentations of SPM and FreeSurfer have major errors in the ventricles and in the area of the thalami and caudate. SPM probability maps were thresholded at $p=0.4$ which was the optimal choice to reduce overlap between tissues and to reduce false positives. 


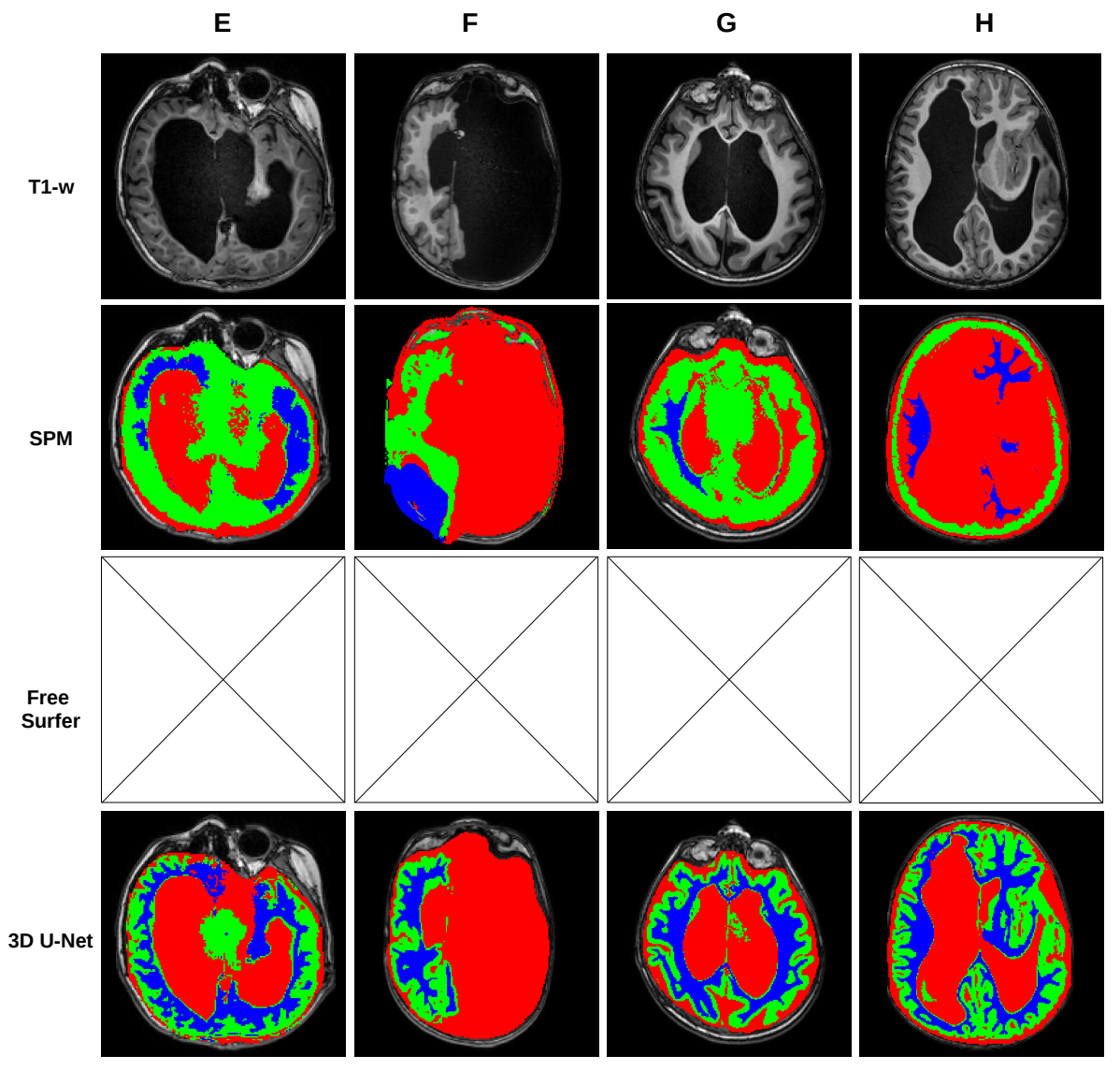

This is an extension of Figure 2, i.e. paradigmatic segmentations of patients with complex cerebral distortions, where we show SPM and FreeSurfer that we excluded in the manuscript, together with the 3D U-Net for comparison. SPM has major macroscopic errors almost everywhere. FreeSurfer failed to converge on the images of these subjects so the related entries are empty, as reported in Section 4. SPM probability maps were thresholded at $p=0.4$ which was the optimal choice to reduce overlap between tissues and to reduce false positives. 